\section{On Symbol Versus Bit Interleaving for Block-Coded Binary Markov Channels}

\author{
Haider Al-Lawati, Fady Alajaji, Senior Member, IEEE, and \\ Cecilio Pimentel
}

\begin{abstract}
We examine bit- and symbol-interleaving strategies for linear nonbinary block codes (under bounded-distance decoding) over the family of binary additive noise finite-state Markov channel (FSMC) models with memory. We derive a simple analytical sufficient condition under which perfect (i.e., with infinite interleaving depth) symbol interleaving outperforms perfect bit interleaving in terms of the probability of codeword error (PCE). It is shown that the well-known Gilbert-Elliott channel (GEC) with positive noise-correlation coefficient and the recently introduced Markovian queue-based channel (QBC) of memory $M$ satisfy this condition. This result has widely been illustrated numerically (without proof) in the literature, particularly for the GEC. We also provide examples of binary FSMC models for which the reverse result holds, i.e., perfect bit interleaving outperforming perfect symbol interleaving. Finally, a numerical PCE study of imperfect symbol-interleaved nonbinary codes over the QBC indicates that there is a linear relationship between the optimal interleaving depth and a function of a single parameter of the QBC.
\end{abstract}

Index Terms-Additive noise channels, binary finite-state Markov channels (FSMCs), Gilbert-Elliott channel (GEC), Markovian queue-based channel (QBC), Reed-Solomon (RS) and nonbinary block codes, symbol and bit interleaving.

\section{INTRODUCTION}

An important class of nonbinary error-correcting codes widely used in data-transmission and storage systems is the family of Reed-Solomon (RS) codes [1]. A commonly used strategy to employ an RS code to correct errors generated by a channel with (statistical) memory is to incorporate block interleaving into the communication system. It is also known that binary-modulated time-correlated flatfading channels used in conjunction with hard-decision demodulation can be represented by stationary binary (modulo-2) additive noise channels with memory (e.g., see [2] and [3]). When nonbinary codewords are sent over such channels, two interleaving strategies are worth considering [4], [5]: 1) interleaving the code symbols and 2) interleaving the code (or channel) bits, which, under perfect or infinite interleaving depth, reduces the channel to the memoryless binary symmetric channel (BSC) [6].

In prior works, the performance of noninterleaved RS codes over correlated fading channels is analyzed in [7]-[9], using a two-step procedure. First, a binary finite-state Markov channel (FSMC) model is introduced for the generation of the bit- or symbol-error process, and then, a formula for the probability of codeword error (PCE) under bounded-distance decoding is derived for the proposed model.

Manuscript received September 22, 2009; revised December 23, 2009; accepted January 31, 2010. Date of publication February 17, 2010; date of current version June 16, 2010. This paper was presented in part at the IEEE Pacific Rim Conference on Communications, Computers, and Signal Processing, Victoria, BC, Canada, August 2007. The review of this paper was coordinated by Prof. H.-F. Lu.

H. Al-Lawati and F. Alajaji are with the Department of Mathematics and Statistics, Queen's University, Kingston, ON K7L 3N6, Canada (e-mail: haider@mast.queensu.ca; fady@mast.queensu.ca).

C. Pimentel is with the Department of Electronics and Systems, Federal University of Pernambuco, 50711-970 Recife-PE, Brazil (e-mail: cecilio@ufpe.br).

Digital Object Identifier 10.1109/TVT.2010.2043276
In [7], the channel is modeled via the Gilbert-Elliott channel (GEC) [6], whose parameters are calculated using a simple threshold model. In [8], level-crossing statistics are applied to characterize the fading arrival process and the fading durations, and the PCE is expressed in terms of the probability distribution of the fading durations. In [9], the bit-error process resulting from hard-decision demodulation of binary frequency-shift keying modulated signals over correlated Rician fading channels is modeled via a Fritchman channel [10]. Imperfect (finite-length) symbol interleaving is also considered in [8] and [9]. In a recent work [11], the performance of symbolinterleaved RS codes over fading channels modeled via the GEC has been examined in the context of CDMA2000 Broadcast and Multicast Services.

A numerical study of the superiority of symbol-interleaved (over bit-interleaved) RS codes is given in [4] for the case of slow-fading channels. This result motivated the authors of [8] to consider only symbol interleaving in their investigations. An analytical expression for the PCE of RS codes over binary FSMC models under imperfect bit and symbol interleaving is derived in [5] for two decoding strategies (bounded-distance decoding and error-forecasting decoding). The study conducted in [5] to compare the performance of these two interleaving strategies for the GEC corroborates the superiority of symbol interleaving found in previous numerical studies. However, since there is no known analytical proof in the literature for this result, it is natural to investigate whether perfect symbol interleaving always outperforms perfect bit interleaving for a given class of binary FSMC models or if there exist conditions on the channel parameters under which bit interleaving provides better PCE performance.

In this paper, we analytically investigate the merits of perfect symbol and bit interleaving for linear nonbinary block codes under bounded-distance decoding over the class of binary FSMCs (with additive stationary hidden Markovian noise). This class of FSMC models includes the GEC (which has widely been shown to be a good model for flat-fading channels [2], [3]) and the recently introduced queue-based channel (QBC) [12]. The QBC, which features an $M$ th-order additive Markov noise process generated via a finite queue, has only four parameters (like the GEC), while allowing its memory order to be arbitrarily large. It also offers (unlike the GEC) closedform expressions for the block-transition probability, capacity, and autocorrelation function [12]. Furthermore, it has been shown that the QBC can accurately approximate the GEC [12] and (uncoded and RS-coded) hard-decision-demodulated Rician flat-fading channels [13], [14].

Imperfect (i.e., with finite interleaving depth) interleaving is an important issue in practice. In particular, for nonbinary block codes over the GEC, it was found in [5] that perfect interleaving can be realized when the interleaving depth is a multiple of the channel's average burst length (e.g., the typical interleaving depth needed to achieve perfect symbol and bit interleaving is double and four times the average burst length of the GEC, respectively [5]). Another motivation for this work is to verify if a similar result also holds for the QBC. We provide PCE numerical results when imperfect symbolinterleaved RS codes are sent over the QBC and investigate the choice of the optimal interleaving depth in terms of the parameters of this channel.

The contributions of this paper are summarized as follows: In Proposition 1, we establish a simple explicit condition (in terms of the FSMC noise statistics) under which perfect symbol interleaving results in a lower PCE, compared with perfect bit interleaving for any linear nonbinary block code used over the FSMC with bounded-distance 
decoding. ${ }^{1}$ We analytically show that both the GEC with positive noise correlation coefficient (i.e., with persistent memory [6]) and the QBC satisfy this condition (see Propositions 2 and 3). Interestingly, we note an opposite behavior for the simplified Gilbert channel (SGC) [15] (i.e., the first-order Markov noise channel) when its noise correlation coefficient is negative ${ }^{2}$ (this channel is a special instance of the GEC with oscillatory memory [6]); in this case, we show that perfect bit-interleaved nonbinary codes outperform perfect symbolinterleaved nonbinary codes. We also provide other examples of FSMC models (Fritchman channels with negative noise correlation coefficient), where bit interleaving can be better than symbol interleaving. Hence, for some classes of channels (such as the GEC), the choice of the best interleaving strategy is directly related to the sign of the noise-correlation coefficient. Finally, we conduct a numerical study to analyze the sensitivity of the QBC and interleaving parameters with respect to the PCE. We found that, similarly to the GEC, there is a linear relationship between the optimal interleaving depth and a function of a single parameter of the QBC.

\section{SYSTEM DESCRIPTION}

We consider a coded communication system where nonbinary transmitted symbols, assuming values from the Galois field $\mathrm{GF}\left(2^{b}\right), b \geq 2$, are mapped one-to-one to a binary $b$-tuple and are transmitted across a binary FSMC model. The $k$ th received binary symbol $Y_{k}$ is described by $Y_{k}=X_{k} \oplus Z_{k}, k=1,2, \ldots$, where $\oplus$ denotes addition modulo-2, $X_{k} \in\{0,1\}$ is the $k$ th transmitted symbol, and $Z_{k} \in\{0,1\}$ is the $k$ th channel noise symbol. We assume that the noise process $\left\{Z_{k}\right\}_{k=1}^{\infty}$ is a stationary hidden Markov source and is independent from the transmitted process $\left\{X_{k}\right\}_{k=1}^{\infty}$. Two channel models considered in this paper (one with an $M$ th-order Markovian noise and one with a hidden Markovian noise), which belong to the class of binary FSMC models, are briefly described next.

\footnotetext{
${ }^{1}$ It is worth pointing out that the result in Proposition 1 does not require that the noise process be hidden Markovian (we only need for the noise to be stationary). We, however, restrict it to being hidden Markovian since FSMC models are widely used to model fading channels.

${ }^{2}$ Note that the case of negative noise correlation coefficient can reflect situations involving very fast correlated Rician fading (cf. Remark 1). Even if such fast-fading situations may rarely occur in practice, the fact that a negative noise correlation leads to bit interleaving outperforming symbol interleaving (i.e., the reverse result of Propositions 1-3) is at least of conceptual interest.
}

\section{A. $Q B C$}

The QBC uses a simple approach to model an $M$ th-order Markov noise process via a finite queue [12]. At the $k$ th time, the channel generates a noise output $Z_{k}$ that depends on four parameters, namely, the size of the queue $M$, the channel bit-error rate (BER), $p=$ $\operatorname{Pr}\left(Z_{k}=1\right)$, and correlation parameters $\varepsilon$ and $\alpha$, where $0 \leq \varepsilon<1$, $\alpha \geq 0$. First, one of two parcels (an urn and a queue of size $M$ ) are selected with probability distribution $\{\varepsilon, 1-\varepsilon\}$. If the urn is selected, the model generates an error $\left(Z_{k}=1\right)$ with probability $p$. If the queue is selected, a binary noise symbol is selected with a probability distribution that depends on $M$ and on the parameter $\alpha$ ( $\alpha$ determines the bias for operating on the last cell of the queue of length $M$ and is equal to 1 when $M=1[12])$. The channel-state process $\left\{S_{k}\right\}_{k=-\infty}^{\infty}$, where $S_{k} \triangleq\left(Z_{k}, Z_{k-1}, \ldots, Z_{k-M+1}\right)$ is a homogeneous first-order Markov process with an alphabet of size $2^{M}$ with $2^{M} \times 2^{M}$ transition probability matrix $\mathbf{P}=\left[p_{i j}\right]$ given by [12, eq. (4)] and state stationary distribution column vector $\Pi=\left[\pi_{i}\right]$ given by [12, eq. (5)]. The QBC allows simple closed-form expressions for several statistics. In particular, the channel-noise block probability $\operatorname{Pr}\left(Z_{1}=z_{1}, Z_{2}=\right.$ $\left.z_{2}, \ldots, Z_{n}=z_{n}\right)=\operatorname{Pr}\left(Z^{n}=z^{n}\right)$ is expressed in (1) and (2), shown at the bottom of the page. The noise correlation coefficient Cor for the $\mathrm{QBC}$ is a nonnegative quantity given by

$$
\begin{aligned}
\text { Cor } & =\frac{\mathbf{E}\left[Z_{1} Z_{2}\right]-\mathbf{E}\left[Z_{1}\right] \mathbf{E}\left[Z_{2}\right]}{\mathbf{E}\left[Z_{1}^{2}\right]-\left(\mathbf{E}\left[Z_{1}\right]\right)^{2}} \\
& =\frac{\frac{\varepsilon}{M-1+\alpha}}{1-(M-2+\alpha) \frac{\varepsilon}{M-1+\alpha}}
\end{aligned}
$$

where $\mathbf{E}[\cdot]$ denotes expectation. When $\varepsilon=0$ (Cor $=0)$, the resulting model reduces to the memoryless BSC with crossover probability $p$.

\section{B. GEC}

The GEC is driven by an underlying stationary ergodic two-state Markov chain composed of state 0 , which produces errors with probability $p_{G}$, and state 1 , where errors occur with probability $p_{B}$, where $p_{G}<p_{B}$. The transition probabilities of the Markov chain are $p_{01}=$ $Q$ and $p_{10}=q$, where $0<Q<1$, and $0<q<1$. Mushkin and BarDavid [6] defined the "memory" of the GEC as $\mu=1-q-Q$. If $\mu>0$, the channel has persistent memory, or if $\mu<0$, the channel has oscillatory memory [6]. When $\mu=0$, the model reduces to the memoryless BSC. We define two matrices $\mathbf{P}(0)$ and $\mathbf{P}(1), \mathbf{P}(0)+$ $\mathbf{P}(1)=\mathbf{P}$, where the $(i, j)$ th entry of the matrix $\mathbf{P}(z), z \in\{0,1\}$,

For blocklength $n \leq M$ :

$$
\begin{aligned}
\operatorname{Pr}\left(Z^{n}=z^{n}\right) & =\frac{\prod_{j=0}^{n-d_{1}^{n}-1}\left[j \frac{\varepsilon}{M-1+\alpha}+(1-\varepsilon)(1-p)\right] \prod_{j=0}^{d_{1}^{n}-1}\left[j \frac{\varepsilon}{M-1+\alpha}+(1-\varepsilon) p\right]}{\prod_{j=M-n}^{M-1}\left[1-(\alpha+j) \frac{\varepsilon}{M-1+\alpha}\right]} \\
\text { where } d_{a}^{b} & =z_{b}+z_{b-1}+\cdots+z_{a}\left(d_{a}^{b}=0 \text { if } a>b\right) \text { and } \prod_{j=0}^{a}(\cdot) \triangleq 1 \text { if } a<0 .
\end{aligned}
$$

For blocklength $n \geq M+1$ :

$$
\begin{aligned}
& \operatorname{Pr}\left(Z^{n}=z^{n}\right)=L^{(M)} \prod_{i=M+1}^{n}\left[\left(d_{i-M+1}^{i-1}+\alpha z_{i-M}\right) \frac{\varepsilon}{M-1+\alpha}+(1-\varepsilon) p\right]^{z_{i}} \\
& \times\left\{\left[\left(M-1-d_{i-M+1}^{i-1}\right)+\alpha\left(1-z_{i-M}\right)\right] \frac{\varepsilon}{M-1+\alpha}+(1-\varepsilon)(1-p)\right\}^{1-z_{i}} \\
& \quad \text { where } L^{(M)}=\frac{\prod_{j=0}^{M-1-d_{1}^{M}}\left[j \frac{\varepsilon}{M-1+\alpha}+(1-\varepsilon)(1-p)\right] \prod_{j=0}^{d_{1}^{M}-1}\left[j \frac{\varepsilon}{M-1+\alpha}+(1-\varepsilon) p\right]}{\prod_{j=0}^{M-1}\left[1-(\alpha+j) \frac{\varepsilon}{M-1+\alpha}\right]} .
\end{aligned}
$$


is $\operatorname{Pr}\left(Z_{k}=z, S_{k}=j \mid S_{k-1}=i\right)$. The state stationary distribution vector is $\Pi=\left[\pi_{0}, \pi_{1}\right]^{T}=[q /(q+Q), Q /(q+Q)]^{T}$ (where the superscript $[\cdot]^{T}$ indicates transposition), and the matrices $\mathbf{P}(0)$ and $\mathbf{P}(1)$ are given by

$$
\begin{aligned}
& \mathbf{P}(0)=\left[\begin{array}{cc}
(1-Q)\left(1-p_{G}\right) & Q\left(1-p_{B}\right) \\
q\left(1-p_{G}\right) & (1-q)\left(1-p_{B}\right)
\end{array}\right] \\
& \mathbf{P}(1)=\left[\begin{array}{cc}
(1-Q) p_{G} & Q p_{B} \\
q p_{G} & (1-q) p_{B}
\end{array}\right] .
\end{aligned}
$$

The channel-noise block probability can be expressed in matrix form as

$$
\operatorname{Pr}\left(Z^{n}=z^{n}\right)=\boldsymbol{\Pi}^{T}\left(\prod_{k=1}^{n} \mathbf{P}\left(z_{k}\right)\right) \mathbf{1}
$$

where 1 is a column vector of ones of length 2. For example, an expression for $p_{0} \triangleq \operatorname{Pr}\left(Z_{k}=0\right)$ is

$$
p_{0}=1-\mathrm{BER}=\pi_{0}\left(1-p_{G}\right)+\pi_{1}\left(1-p_{B}\right) .
$$

The noise correlation coefficient for the GEC is expressed as

$$
\text { Cor }=\frac{\mu\left(\operatorname{BER}-p_{G}\right)\left(p_{B}-\mathrm{BER}\right)}{\operatorname{BER}(1-\mathrm{BER})} .
$$

The SGC [15] can be obtained from the GEC by setting $p_{G}=0$ and $p_{B}=1$. As a result, the SGC with BER $p$ and noise correlation coefficient $\mu$ is a two-state first-order Markov noise channel with parameters $Q=(1-\mu) p$ and $q=(1-\mu)(1-p)$. It directly follows from (4) and (5) that Cor and $\mu$ have identical signs.

\section{Perfect Bit Interleaving Versus Perfect Symbol INTERLEAVING FOR NONBINARY CODES}

The objective of this section is to analytically compare the performance of nonbinary codes under both perfect symbol interleaving and perfect bit interleaving when transmitted over the binary FSMC model described at the beginning of the previous section.

Let $\mathcal{C}$ be any nonbinary linear block code over the Galois field $\mathrm{GF}\left(2^{b}\right)$ with length $n$ and error-correction capability $t$ (e.g., an RS code). A transmitted symbol is correctly received if the stationary noise corrupting it is a sequence of zeros of length $b$, which is denoted $0^{b}$. Otherwise, the transmitted symbol is incorrectly received, and a symbol error occurs. Let the probability that the channel produces the $b$-tuple all zeros be denoted by $F(b)=\operatorname{Pr}\left(Z^{b}=0^{b}\right)$. Then, the probability of correct decoding under bounded-distance decoding, which is denoted $P_{c}$, for the perfect symbol-interleaved system, is given by

$$
P_{c}=\sum_{i=0}^{t}\left(\begin{array}{l}
n \\
i
\end{array}\right)(1-F(b))^{i}(F(b))^{n-i} .
$$

On the other hand, for the perfect bit-interleaved nonbinary code, denote the probability of correct $b$ transmissions by $G(b) \triangleq \operatorname{Pr}(Z=0)^{b}$. Hence, the probability of correct decoding for this interleaving scheme is given by (6), replacing $F(b)$ by $G(b)$. The performance comparison carried out in this section is done in terms of $P_{c}$ or, equivalently, in terms of PCE $=1-P_{c}$.

Proposition 1: If $F(b)>G(b)$ for the binary FSMC model, then perfect symbol interleaving outperforms perfect bit interleaving for the transmission of $\mathcal{C}$ under bounded-distance decoding.
Proof $^{3}$ : If $x$ denotes the symbol error probability, then the PCE (under bounded-distance decoding) can be expressed as a function of $x$ as follows:

$$
\operatorname{PCE}(x)=\sum_{i=t+1}^{n}\left(\begin{array}{l}
n \\
i
\end{array}\right) x^{i}(1-x)^{n-i}=I_{x}(t+1, n-t)
$$

where $I_{x}(a, b)$ is the regularized incomplete beta function given by

$$
\begin{aligned}
I_{x}(a, b) & =\frac{1}{B(a, b)} \int_{0}^{x} y^{a-1}(1-y)^{b-1} d y \\
& =\sum_{i=a}^{a+b-1}\left(\begin{array}{c}
a+b-1 \\
i
\end{array}\right) x^{i}(1-x)^{a+b-1-i}
\end{aligned}
$$

for $a>0, b>0$, and $B(a, b)=\int_{0}^{1} y^{a-1}(1-y)^{b-1} d y$ is the beta function and is positive for all positive pairs $(a, b)$. The function $I_{x}(a, b)$ is monotonically increasing with respect to $x$ in the range $0<x<1$, since

$$
\frac{d I_{x}(a, b)}{d x}=\frac{x^{a-1}(1-x)^{b-1}}{B(a, b)}>0 .
$$

Therefore, the PCE under perfect symbol interleaving, i.e., PCE $(1-$ $F(b)$ ), is smaller than the PCE under perfect bit interleaving, i.e., $\operatorname{PCE}(1-G(b))$, whenever $F(b)>G(b)$.

In light of Proposition 1, we next show that perfect symbol interleaving is always better compared with perfect bit interleaving when the nonbinary code is transmitted over either the QBC or the GEC with positive memory.

\section{A. $Q B C$}

Proposition 2: Under bounded-distance decoding, perfect symbol interleaving outperforms perfect bit interleaving when nonbinary codes over $\mathrm{GF}\left(2^{b}\right)$ are transmitted over the $\mathrm{QBC}$, for $\varepsilon>0$ and $p>0$.

Proof: From Proposition 1, it is enough to show that $F(b)>$ $G(b)$ for the QBC. For this channel, $G(b)=(1-p)^{b}$, and for $b \leq M$, we express $F(b)$ using (1) as

$$
F(b)=\prod_{j=0}^{b-1} \frac{j \frac{\varepsilon}{M-1+\alpha}+(1-\varepsilon)(1-p)}{1-(\alpha+M-1-j) \frac{\varepsilon}{M-1+\alpha}} .
$$

For each $j>0$, we notice that, for $p>0$

$$
\frac{j \frac{\varepsilon}{M-1+\alpha}+(1-\varepsilon)(1-p)}{1-(\alpha+M-1-j) \frac{\varepsilon}{M-1+\alpha}}>(1-p) .
$$

Because $b>1$ (for nonbinary codes), we get

$$
\prod_{j=0}^{b-1} \frac{j \frac{\varepsilon}{M-1+\alpha}+(1-\varepsilon)(1-p)}{1-(\alpha+M-1-j) \frac{\varepsilon}{M-1+\alpha}}>(1-p)^{b}
$$

which implies that $F(b)>G(b)$. When $b>M, F(b)$ is expressed using (2) as

$$
\begin{aligned}
& F(b)=\prod_{j=0}^{M-1} \frac{j \frac{\varepsilon}{M-1+\alpha}+(1-\varepsilon)(1-p)}{1-(\alpha+M-1-j) \frac{\varepsilon}{M-1+\alpha}} \\
& \times(\varepsilon+(1-\varepsilon)(1-p))^{b-M} .
\end{aligned}
$$

\footnotetext{
${ }^{3}$ This proof, which is based on expressing the PCE (which is the binomial complementary cumulative-distribution function) in terms of the regularized incomplete beta function, is due to one of the anonymous reviewers (Reviewer 1). We herein include it in lieu of our original proof as it is simpler.
} 
We already remarked that $(j(\varepsilon /(M-1+\alpha))+(1-\varepsilon)(1-p)) /$ $(1-(\alpha+M-1-j)(\varepsilon /(M-1+\alpha)))>(1-p)$ for $j>0$. We also note that

$$
\varepsilon+(1-\varepsilon)(1-p)=(1-p)+\varepsilon p \geq(1-p)
$$

with equality if and only if either $p=0$ or $\varepsilon=0$. Therefore, we combine the aforementioned two inequalities to get that

$$
\begin{gathered}
\left(\prod_{j=0}^{M-1} \frac{j \frac{\varepsilon}{M-1+\alpha}+(1-\varepsilon)(1-p)}{1-(\alpha+M-1-j) \frac{\varepsilon}{M-1+\alpha}}(\varepsilon+(1-\varepsilon)(1-p))^{b-M}\right) \\
>(1-p)^{M}(1-p)^{b-M}=(1-p)^{b} .
\end{gathered}
$$

Therefore, $F(b)>G(b)$ (the inequality is strict because we assume that both $p$ and $\varepsilon$ are not equal to zero).

\section{B. $G E C$}

For the GEC model, $G(b)=p_{0}^{b}$, where $p_{0}$ is given by (4). We do not derive an explicit expression for $F(b)$. Alternatively, we define the generating series for $F(b)$ as

$$
\mathcal{F}(z) \triangleq \sum_{b=0}^{\infty} F(b) z^{b} .
$$

It follows from (3) that $F(b)=\boldsymbol{\Pi}^{T} \mathbf{P}^{b}(0) \mathbf{1}$. Then [16]

$$
\mathcal{F}(z)=\boldsymbol{\Pi}^{T}(\mathbf{I}-\mathbf{P}(0) z)^{-1} \mathbf{1}
$$

where $\mathbf{I}$ is the identity matrix. For the GEC, $\mathcal{F}(z)$ in (7) becomes

$$
\mathcal{F}(z)=\frac{1+a_{1} z}{1+b_{1} z+b_{2} z^{2}}
$$

where

$$
\begin{aligned}
& a_{1}=-\mu\left[\pi_{1}\left(1-p_{G}\right)+\pi_{0}\left(1-p_{B}\right)\right] \\
& b_{1}=-\left[(1-\mu) p_{0}+\mu\left(2-p_{G}-p_{B}\right)\right] \\
& b_{2}=\mu\left(1-p_{G}\right)\left(1-p_{B}\right) .
\end{aligned}
$$

The following recursion formula is directly derived from (8):

$$
F(b)=-b_{1} F(b-1)-b_{2} F(b-2)
$$

for $b \geq 2$, with initial conditions $F(0)=1$ and $F(1)=p_{0}$. The condition stated in Proposition 1 holds for the GEC in light of the next lemma.

Lemma 1: The following relation is satisfied for the GEC with $\mu>0$ :

$$
\frac{F(b)}{F(b-1)}>p_{0}, \quad \text { for } b \geq 2 .
$$

Proof: The proof is by induction on $b$. For $b=2$, the expressions for $p_{0}$ and $p_{00} \triangleq \operatorname{Pr}\left(Z_{k}=0, Z_{k+1}=0\right)$ calculated from (3) yield

$$
\frac{F(2)}{F(1)}=\frac{p_{00}}{p_{0}}=-b_{1}-\frac{b_{2}}{p_{0}}=p_{0}+\mu \frac{\pi_{0} \pi_{1}\left(p_{B}-p_{G}\right)^{2}}{p_{0}}>p_{0}
$$

since $\mu>0$. Next, assume that the statement (10) is true for a fixed $b \geq 2$. It follows from (9) that

$$
F(b+1)=-b_{1} F(b)-b_{2} F(b-1)
$$

or

$$
\frac{F(b+1)}{F(b)}=-b_{1}-b_{2} \frac{F(b-1)}{F(b)} .
$$

We conclude from the inductive hypothesis that $F(b-1) / F(b)<$ $1 / p_{0}$, and since $b_{2}>0$ for $\mu>0$, we obtain that

$$
\frac{F(b+1)}{F(b)}>-b_{1}-\frac{b_{2}}{p_{0}}=\frac{F(2)}{F(1)}>p_{0} .
$$

By repeatedly using (10) for increasing values of $b$, we obtain a chain of inequalities of the form $F(b)>F(b-x) p_{0}^{x}$. In particular, when $x=$ $b, F(b)>G(b)$. Thus, we have proved the following proposition.

Proposition 3: Perfect symbol-interleaved transmission of $\mathcal{C}$ performs better than perfect bit-interleaved transmission over the GEC with $\mu>0$, assuming bounded-distance decoding.

\section{Channels With Negative Noise-Correlation Coefficient}

We next observe that, for some classes of FSMC models with negative noise-correlation coefficient, perfect bit interleaving can be better than perfect symbol interleaving.

Remark 1: The noise correlation coefficient of a communication fading system is generally (but not always) a positive quantity. To illustrate this, let us model (using the fitting method of [13]) via a GEC a discrete channel with binary frequency-shift keying modulation, Rician fading with Clarke's autocorrelation function, and hard quantized noncoherent demodulation [13]. For the case of Rayleigh fading, the correlation coefficient of this discrete channel is always nonnegative. However, when the fading is Rician, there exists a range of fading parameters that yield a GEC with negative memory $\mu$ (or negative noise correlation coefficient). For example, for a discrete fading channel with an SNR of $13 \mathrm{~dB}$, a normalized Doppler frequency of $f_{D} T=0.6$, and a Rician factor of $K_{R}=3 \mathrm{~dB}$, we obtain a fitting GEC with parameters $p_{G}=0.0014, p_{B}=0.06, q=0.923$, and $Q=$ 0.6175 . The resulting BER is 0.025 , and $\mu=-0.54$; in addition, the capacity values of the GEC and the (equivalent) BSC (under perfect bit interleaving) are 0.8323 and 0.8319 bits/channel use, respectively.

Remark 2: Note that, in Proposition 1, if $F(b)<G(b)$, then we get the opposite result compared with the positive noise-correlation case, i.e., perfect bit interleaving outperforms perfect symbol interleaving. For the SGC, $F(b)$ and $G(b)$ are given by

$$
\begin{aligned}
& F(b)=\left[(1-p)(\mu+(1-\mu)(1-p))^{b-1}\right] \\
& G(b)=\left[(1-p)^{b}\right] .
\end{aligned}
$$

Note that, if $\mu<0$, then $F(b)<G(b)$.

Remark 3: Finally, note that we can construct examples of a simplified Fritchman channel [10] of negative noise-correlation coefficient and with two good states and one bad state such that $F(2)<G(2)$ but $F(3)>G(3) .{ }^{4}$ Thus, for this channel, neither perfect symbol interleaving nor perfect bit interleaving is always better, since this comparison depends on the code's field size $2^{b}$.

Propositions 1-3 consider the PCE performance of nonbinary codes under perfect interleaving. The next section provides a practical guideline to design the optimal interleaving depth (e.g., the typical interleaving depth needed to achieve perfect interleaving) for the QBC.

\section{Performance Evaluation Under IMPERFECT INTERLEAVING}

In this section, we conduct a numerical PCE study of imperfect interleaved nonbinary block codes (under bounded-distance decoding)

\footnotetext{
${ }^{4}$ Using the notation of [10], consider, for example, a Fritchman channel with parameters $p_{11}=0.11, p_{22}=0.82, p_{31}=0.42$, and $p_{32}=0.3$; its noise correlation coefficient is -0.057 . For this channel, we have $F(2)<G(2)$ and $F(3)>G(3)$.
} 


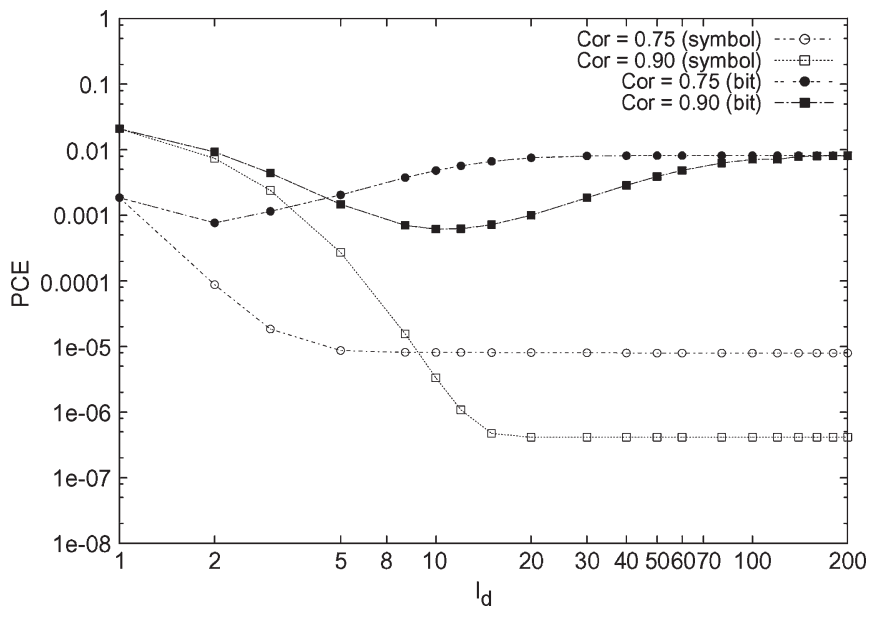

Fig. 1. PCE versus $I_{d}$ for $(73,57) \mathrm{RS}, b=7$, and $t=8$ over the QBC with parameters $M=2, \alpha=1, p=0.007$, and Cor $=0.75,0.90$. Symbol and bit interleaving.

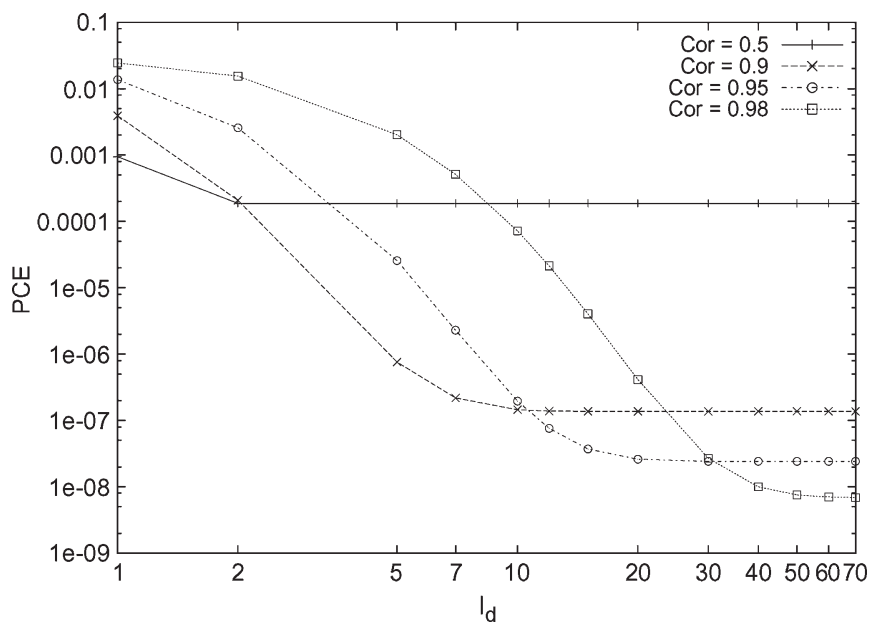

Fig. 2. PCE versus $I_{d}$ for $(73,57) \mathrm{RS}, b=7$, and $t=8$ over the QBC with parameters $M=1, \alpha=1, p=0.007$, and $\mathrm{Cor}=0.5,0.9,0.95,0.98$. Symbol interleaving.

over the QBC. The performance is evaluated via the derivation of the probability of $m$ errors in a block of length $n$, namely, $P(m, n)$, yielding a PCE given by $\mathrm{PCE}=\sum_{m=t+1}^{n} P(m, n)$. For our purposes, we numerically calculate $P(m, n)$ and the PCE using the method of [9]; however, the recent analytical method of [5] can also be used. We consider an $(n, k)$ RS code over $\mathrm{GF}\left(2^{b}\right)$ with codewords of length $n$ and $k$ information symbols. We assume block symbol interleaving with $n b$ columns (codeword length in bits) and $I_{d}$ (interleaving depth) rows. The $b$ bits within each symbol are consecutively transmitted through the channel.

The superiority of imperfect symbol-interleaved nonbinary codes to imperfect bit-interleaved nonbinary codes over the GEC was observed in [5]. Similar results can be obtained for the QBC (see, for example, Fig. 1). This figure presents PCE versus $I_{d}$ for a bit- and symbolinterleaved shortened $(73,57) \mathrm{RS}$ code (with $b=7, t=8$ symbols) over the QBC with parameters $M=2, \alpha=1, p=0.007$, and two values of Cor, i.e., Cor $=0.75,0.9$. We observe that imperfect symbol interleaving outperforms imperfect bit interleaving for all values of $I_{d}$. In particular, for sufficiently large $I_{d}$, these curves corroborate the result presented in Proposition 2. Motivated by these results, we hereafter focus on symbol interleaving, and our objective is to investigate the existence of a relationship between the optimal interleaving depth and the QBC parameters.
TABLE I

OPTIMAL INTERLEAVING DEPTH FOR THE QBC WITH PARAMETERS $M=1, \alpha=1, p=0.007$

\begin{tabular}{|c|c|}
\hline$\varepsilon$ & $I_{d}^{\star}$ \\
\hline 0.5 & 2 \\
0.9 & 10 \\
0.95 & 20 \\
0.98 & 50 \\
\hline
\end{tabular}

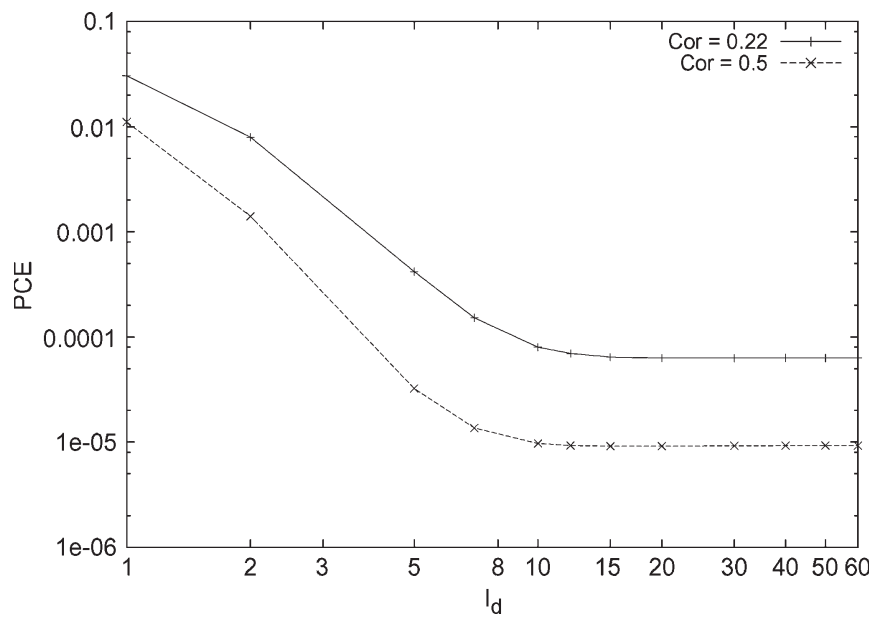

Fig. 3. PCE versus $I_{d}$ for $(73,57) \mathrm{RS}, b=7$, and $t=8$ over the QBC with parameters $M=4, \varepsilon=0.8, p=0.007$, and Cor $=0.22,0.5$. Symbol interleaving.

Fig. 2 presents PCE versus $I_{d}$ for a symbol-interleaved shortened $(73,57) \mathrm{RS}$ code (with $b=7, t=8$ symbols) over the QBC. The parameters of the $\mathrm{QBC}$ are $M=1(\alpha=1), p=0.007$, and four values of $\operatorname{Cor}($ or $\varepsilon$ ), i.e., Cor $=0.5,0.9,0.95,0.98$. For a given value of Cor, we observe that the PCE decreases as $I_{d}$ increases until a threshold point at which it is no longer possible to improve the PCE. We denote this value of $I_{d}$ that renders the channel block memoryless (i.e., achieving perfect symbol interleaving) by $I_{d}^{\star}$. The approximate values of $I_{d}^{\star}$ found from each curve of this figure are listed in Table I. We notice from this table a linear relationship between $I_{d}^{\star}$ and $1 /(1-\varepsilon)$, which is expressed as $I_{d}^{\star}=1 /(1-\varepsilon)$. We conduct in the following a similar analysis for a QBC with higher memory orders $M$.

Fig. 3 presents PCE versus $I_{d}$ for a symbol-interleaved shortened (73, 57) RS code over the QBC with $M=4, \varepsilon=0.8, p=0.007$, and two values of Cor, i.e., Cor $=0.22(\alpha=11.2), 0.5(\alpha=1)$. The values of $I_{d}^{\star}$ are roughly the same for each curve, which allow us to conclude that for a fixed $\varepsilon, I_{d}^{\star}$ is weakly dependent on the parameter $\alpha$. A similar conclusion can be derived for the parameter $p$ (curves not shown). We now fix $\alpha=1$ and $p=0.007$ and plot in Fig. 4 the PCE versus $I_{d}$ for a QBC with $M=4$ and Cor $=$ $0.2(\varepsilon=0.5), 0.5(\varepsilon=0.8), 0.69(\varepsilon=0.9)$, and $0.83(\varepsilon=0.95)$. A similar curve is presented in Fig. 5 for $M=6$ and Cor $=$ $0.14(\varepsilon=0.5), 0.4(\varepsilon=0.8), 0.6(\varepsilon=0.9)$, and $0.76(\varepsilon=0.95)$. The values of $I_{d}^{\star}$ achieved for each $\varepsilon$ in these figures are shown in Table II, which can be expressed as $I_{d}^{\star}=\Gamma / 1-\varepsilon$, where $\Gamma=1.5$ for $M=4$ and $\Gamma=2.0$ for $M=6$. Thus, for fixed $(\alpha, p, M)$, a linear relationship between $I_{d}^{\star}$ and $1 /(1-\varepsilon)$ is valid for the $\mathrm{QBC}$, where the proportional constant $\Gamma$ increases with $M$. The same trend is observed for other values of code parameters (figures are not herein shown due to space limitations). This result provides the communication system designer with some insight for the practical 


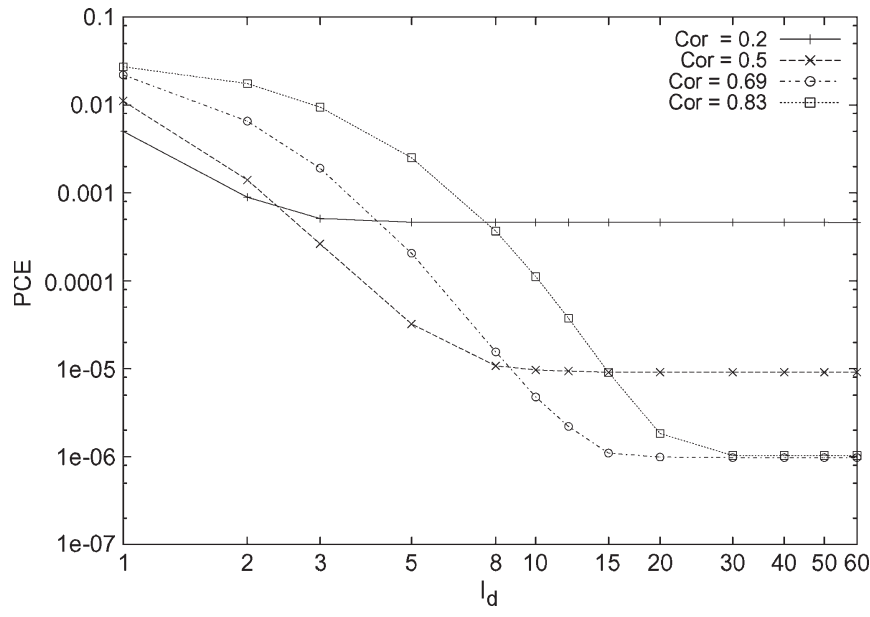

Fig. 4. PCE versus $I_{d}$ for $(73,57) \mathrm{RS}, b=7$, and $t=8$ over the QBC with parameters $M=4, \alpha=1, p=0.007$, and Cor $=0.2,0.5,0.7,0.83$. Symbol interleaving.

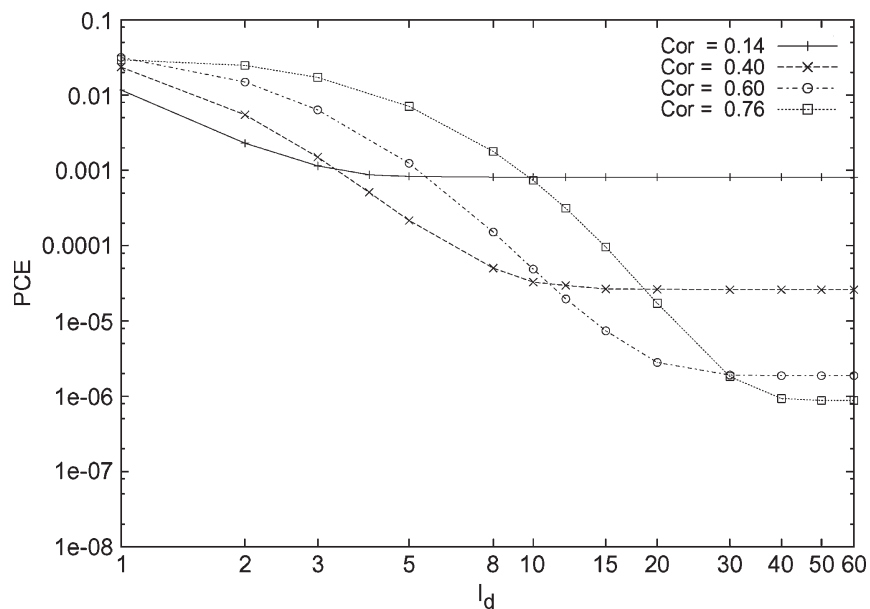

Fig. 5. PCE versus $I_{d}$ for $(73,57) \mathrm{RS}, b=7$, and $t=8$ over the QBC with parameters $M=6, \alpha=1, p=0.007$, and Cor $=0.14,0.4,0.6,0.76$. Symbol interleaving.

TABLE II

OPTIMAL INTERLEAVING DEPTH FOR THE QBC WITH $M=4$ AND $M=6$ DERIVED FROM FIGS. 4 AND 5

\begin{tabular}{|c|c|c|}
\hline$\varepsilon$ & $I_{d}^{\star}(M=4)$ & $I_{d}^{\star}(M=6)$ \\
\hline 0.5 & 3 & 4 \\
0.8 & 8 & 10 \\
0.9 & 15 & 20 \\
0.95 & 30 & 40 \\
\hline
\end{tabular}

interleaving design for the QBC. For example, in a recent work [14], QBC models at the packet level have been developed for a noninterleaved RS-coded communication system with a time-correlated flat-fading channel. For $f_{D} T=0.0005, \mathrm{SNR}=15 \mathrm{~dB}$, and Rayleigh fading, an accurate QBC has parameters $M=4$ and $\varepsilon=0.8773$ (cf. [14, Table I]). The results of this section indicate that $I_{d}^{\star}=12$ for this QBC.

\section{CONCLUSION}

In this paper, we have mathematically demonstrated that for a class of binary additive noise finite-state channels satisfying an explicit (sufficient) condition expressed in terms of the channel-noise statistics, perfectly interleaving the channel at the (code) symbol level always outperforms perfectly interleaving it at the bit level when transmitting nonbinary linear block codes over such channels. We have shown that the GEC with positive noise correlation and the recently introduced Markovian QBC are two finite-state channels for which the condition holds. Both of these channels have previously been shown to accurately model hard-decision-demodulated time-correlated Rayleigh fading channels and slow-fading Rician channels (e.g., see [3] and [13]). Furthermore, we have remarked that there exist finite-state channels (such as the GEC and Fritchman channels with negative noise correlations, which can model Rician channels with fast fading) for which a reverse result holds, i.e., for which bit interleaving outperforms symbol interleaving. Finally, we have conducted a numerical study to evaluate the effects of finite-length (imperfect) symbol interleaving on the performance of RS codes sent over the QBC. We observe that, as for the case of the GEC [5], there exists a simple linear relationship between the optimal interleaving depth and a function of a channelcorrelation parameter; such a property provides useful interleaving design criteria when operating over the QBC and the underlying fading channels it represents.

\section{ACKNOWLEDGMENT}

The authors would like to thank all the anonymous reviewers for their constructive comments, which helped improve this paper, particularly Reviewer 1, who has indicated an alternative proof for Proposition 1 that is simpler than the proof that the authors originally presented.

\section{REFERENCES}

[1] S. Lin and D. Costello, Jr., Error Control Coding: Fundamentals and Applications, 2nd ed. Englewood Cliffs, NJ: Prentice-Hall, 2004.

[2] L. Wilhelmsson and L. B. Milstein, "On the effect of imperfect interleaving for the Gilbert-Elliott channel," IEEE Trans. Commun., vol. 47, no. 5, pp. 681-688, May 1999.

[3] C. Pimentel, T. H. Falk, and L. Lisbôa, "Finite-state Markov modeling of correlated Rician-fading channels," IEEE Trans. Veh. Technol., vol. 53, no. 5, pp. 1491-1501, Sep. 2004.

[4] S. B. Wicker, "Reed-Solomon error control coding for Rayleigh fading channels with feedback," IEEE Trans. Veh. Technol., vol. 41, no. 2, pp. 124-133, May 1992.

[5] K. Sakakibara and J. Yamakita, "Performance comparison of imperfect symbol- and bit-interleaving of block codes over $\operatorname{GF}\left(2^{m}\right)$ on a Markovian channel," IEEE Trans. Wireless Commun., vol. 3, no. 1, pp. 269-277, Jan. 2004.

[6] M. Mushkin and I. Bar-David, "Capacity and coding for the GilbertElliott channel," IEEE Trans. Inf. Theory, vol. 35, no. 6, pp. 1277-1290, Nov. 1989

[7] H. Labiod, "Performance of Reed Solomon error-correcting codes on fading channels," in Proc. IEEE Int. Conf. Pers. Wireless Commun., 1999, pp. 259-263.

[8] J. Lai and N. Mandayam, "Performance of Reed-Solomon codes for hybrid-ARQ over Rayleigh fading channels under imperfect interleaving," IEEE Trans. Commun., vol. 48, no. 10, pp. 1650-1659, Oct. 2000.

[9] C. Pimentel and I. F. Blake, "Concatenated coding performance for FSK modulation on time-correlated Rician fading channels," IEEE Trans. Commun., vol. 46, no. 12, pp. 1610-1618, Dec. 1998.

[10] B. D. Fritchman, "A binary channel characterization using partitioned Markov chains," IEEE Trans. Inf. Theory, vol. IT-13, no. 2, pp. 221-227, Apr. 1967.

[11] K. Kang, "Probabilistic analysis of data interleaving for Reed-Solomon coding in BCMCS," IEEE Trans. Wireless Commun., vol. 7, no. 10, pp. $3878-3888$, Oct. 2008

[12] L. Zhong, F. Alajaji, and G. Takahara, "A binary communication channel with memory based on a finite queue," IEEE Trans. Inf. Theory, vol. 53, no. 8, pp. 2815-2840, Aug. 2007.

[13] L. Zhong, F. Alajaji, and G. Takahara, "A model for correlated Rician fading channels based on a finite queue," IEEE Trans. Veh. Technol., vol. 57, no. 1, pp. 79-89, Jan. 2008. 
[14] C. Pimentel and F. Alajaji, "Packet-based modeling of Reed-Solomon block coded correlated fading channels via a Markov finite queue model," IEEE Trans. Veh. Technol., vol. 58, no. 7, pp. 3124-3136, Sep. 2009.

[15] J. Yee and E. Weldon, "Evaluation of the performance of error-correcting codes on a Gilbert channel," IEEE Trans. Commun., vol. 43, no. 8, pp. 2316-2323, Aug. 1995.

[16] W. Turin, Performance Analysis of Digital Transmission Systems. New York: Comput. Sci., 1990.

\section{Blind Near-MAP Selection Diversity With PIC-LSP Multiuser Detection for Multiuser OFDM}

\author{
Yu-Yi Cheng, Member, IEEE, Yumin Lee, Senior Member, IEEE, \\ and Hsueh-Jyh Li, Senior Member, IEEE
}

\begin{abstract}
In this paper, a receiver algorithm for uplink multiuser orthogonal frequency-division multiplexing (OFDM) with a receiverdiversity system is proposed. The receiver uses a simple multiuser generalization of the maximum a posteriori probability (MAP) selection diversity combining followed by parallel interference cancellation with least-squares projection (PIC-LSP) for multiuser detection. Compared with the nearmaximal-ratio-combining (near-MRC) scheme, simulation results show that the proposed approach achieves very good performance and has much lower complexity.
\end{abstract}

Index Terms-Least-squares projection (LSP), multiuser detection, near-maximum a posteriori probability (MAP) selection diversity, orthogonal frequency-division multiplexing (OFDM), parallel interference cancellation (PIC).

\section{INTRODUCTION}

Orthogonal frequency-division multiplexing (OFDM) is an efficient transmission technique for high-rate wireless data-communication systems. In OFDM, a high-rate data stream is transmitted over lowrate parallel subcarriers. Thus, the symbol duration increases, and the effect of multipath delay spread caused by the multipath propagation characteristic of the channel can be reduced. However, all subcarriers transmitted in a multipath fading channel will arrive at the receiver with different amplitudes. Some subcarriers may be completely lost because of deep fade [1]. Furthermore, in a multiuser scenario where many users share the same set of subcarriers, OFDM also suffers from interference. Receiver diversity [2], including maximal ratio combining (MRC), equal-gain combining (EGC), and selection diversity combining (SDC), is a technique for mitigating both effects. For these techniques, in general, the performance of MRC is superior to EGC and SDC.

Many multiuser detection techniques are widely used in multiuser communication systems. For the interference cancellation technique, in general, the computation procedure can be implemented by iterative or multistage detection because it is decision driven. Most of interference cancellation can be categorized into two classes, namely, successive interference cancellation (SIC) and parallel interference

Manuscript received February 17, 2009; revised September 7, 2009 and December 17, 2009; accepted February 14, 2010. Date of publication March 15, 2010; date of current version June 16, 2010. The review of this paper was coordinated by Prof. U. Tureli.

Y.-Y. Cheng is with the Industrial Technology Research Institute, Hsinchu 31040, Taiwan (e-mail: docheng1129@yahoo.com.tw).

Y. Lee and H.-J. Li are with the National Taiwan University, Taipei 10617, Taiwan (e-mail: yuminlee@ieee.org; hjli@ew.ee.ntu.edu.tw).

Digital Object Identifier 10.1109/TVT.2010.2045776 cancellation (PIC) [3], [4]. The difference between SIC and PIC is that PIC uses temporary decisions of all users to estimate the interference of each user at one iteration or stage. For SIC and PIC, the reliability of the previous decision will affect the result of the next decision. In general, if the signal power for all users is quite different, SIC outperforms PIC. Otherwise, PIC has better performance because this cancellation operation can cancel simultaneously the interference.

In [5], we generalize the maximum a posteriori probability (MAP) SDC scheme proposed in [6] to a multiuser OFDM diversity receiver and propose the multiuser detection algorithm, i.e., SIC with leastsquares projection (SIC-LSP), to detect the transmitted signals of all users. In this paper, we propose another MAP SDC scheme for a multiuser OFDM diversity receiver. There are several features in the proposed receiver. First, a simple approximate MAP SDC scheme is performed on a per-subcarrier basis using novel selection metrics. For per-subcarrier signal processing, this method can be regarded as a simple single-carrier SDC scheme. Second, to compute the selection metrics and detect the information bits for each user, the channel estimates are required. The multiuser blind channel estimation algorithm proposed in [7] is used to derive the channel estimates between all users and receiver antennas. Third, after per-subcarrier SDC is completed, the transmitted signals from each user are separated using a new recursive algorithm, which is referred to as PIC-LSP. Finally, the receiver wherein no pilots are required at all is blind. With the same blind channel estimates and the same number of receiver antennas, simulation results show that the performance for a new proposed MAP SDC scheme with much lower complexity is closely to per-subcarrier basis MRC.

\section{System ModeL}

Consider a multiuser OFDM transmission system shown in Fig. 1, in which $S$ quasi-synchronous users transmit independent and identically distributed OFDM symbols in the same bandwidth at the same time. For the $s$ th user, information bits are encoded and mapped onto quadrature phase-shift keying (QPSK) subsymbols with symbol energy $\sigma_{s}^{2}$. The QPSK subsymbols are serial-to-parallel converted into blocks of $N$ and processed by the $N$-point inverse discrete Fourier transformer (IDFT). The output of the $N$-point IDFT is parallel-toserial converted, and a cyclic prefix (CP) of length $N_{\mathrm{CP}}$ is inserted to obtain a discrete-time signal $x_{s, n}, n=0,1,2 \ldots$ The continuoustime transmitted signal of user $s$ is given by

$$
x_{s}(t) \equiv \sum_{n} x_{s, n} q(t-n T)
$$

where $T$ is the length of one OFDM subsymbol, $n$ is the discrete-time signal index, and $q(\cdot)$ is the pulse-shaping function. The user signals are transmitted to $M$ receiving antennas through independent wireless channels, each modeled as a modified Jakes' frequency-selective multipath fading channel corrupted by additive white Gaussian noise (AWGN) [8]. Here, the new modified Jakes' fading channel model shown in [8] can produce uncorrelated fading waveforms. The statistics of the modified Jakes' fading model (moment and correlation expressions) are shown in [9].

At the receiver, the signals $u^{(m)}(t)$ received from the $m$ th antenna $(m=0, \ldots, M-1)$ are each filtered and sampled at a rate of $P / T$ samples per second, resulting in a discrete-time signal given by

$$
\begin{array}{r}
u_{n, p}^{(m)} \equiv u^{(m)}\left(n T+\frac{p T}{P}\right)=\sum_{s=0}^{S-1} \sum_{l=0}^{L-1} x_{s, n-l} h_{l, s, p}^{(m)}+v_{n, p}^{(m)} \\
m=0,1, \ldots, M-1 p=0,1, \ldots, P-1 n=0,1,2, \ldots
\end{array}
$$

